\title{
Vaccine Informatics
}

\author{
Yongqun He, ${ }^{1}$ Rino Rappuoli, ${ }^{2}$ Anne S. De Groot, ${ }^{3,4}$ and Robert T. Chen ${ }^{5}$ \\ ${ }^{1}$ Unit for Laboratory Animal Medicine, Department of Microbiology and Immunology, Center for Computational Medicine and \\ Bioinformatics and Comprehensive Cancer Center, University of Michigan Medical School, Ann Arbor, MI 48109, USA \\ ${ }^{2}$ Novartis Vaccines and Diagnostics, 53100 Siena, Italy \\ ${ }^{3}$ EpiVax, Inc., Providence, RI 02903, USA \\ ${ }^{4}$ Institute for Immunology and Informatics, University of Rhode Island, Providence, RI 02903, USA \\ ${ }^{5}$ HIV Vaccine and Special Studies Team, Centers for Disease Control and Prevention (CDC/DHAP/EB), Atlanta, GA 30333, USA
}

Correspondence should be addressed to Yongqun He, yongqunh@umich.edu

Received 31 December 2010; Accepted 31 December 2010

Copyright ( $) 2010$ Yongqun He et al. This is an open access article distributed under the Creative Commons Attribution License, which permits unrestricted use, distribution, and reproduction in any medium, provided the original work is properly cited.

Global public health has dramatically increased due to the successful and effective implementation of immunization programs that utilize major infectious disease vaccines. Among the most famous vaccines are Jenner's smallpox vaccine, Pasteur's rabies and anthrax vaccines, and the Bacillus Calmette-Guérin (or Bacille Calmette-Guérin, BCG) vaccine for tuberculosis. The positive effect of vaccination on public health is highlighted by the success of the global smallpox eradication campaign, and the scope of vaccine development for the prevention and treatment of various diseases in both humans and animals continues to grow on a daily basis.

Vaccine informatics is an emerging field that develops and applies computational, statistical, and bioinformatics methods to study vaccine and vaccination-related issues in different stages of research, development, clinical trial, postlicensure clinical uses, and surveillance. The field began centuries ago with widespread vaccination monitoring to determine vaccine safety and clinical applicability. Yet it was not until the growth of the immunoinformatics field in the 1980s that vaccine informatics emerged as an important area of study, when many methods were developed and implemented to predict T-cell and B-cell immune epitopes. These T-cell and B-cell immune epitopes were identified as potential vaccine targets and were recognized as crucial to understanding basic protective immunity. In the 1990s, bioinformatics became an emerging science after more and more DNA, RNA, and protein sequences were identified and studied. In the postgenomics and information era, the application of bioinformatics tools within the vaccinology field has fostered dramatic progression for both literature and data on vaccine informatics, creating the potential to revolutionize every aspect of the pre- and postlicensure vaccine enterprise.

Due to the differing data types available, separate bioinformatics technologies have been developed and applied to address specific vaccine-related issues.

(1) Immune epitope data: an immune epitope is a part of an antigen recognized by antibodies, B cells, or T cells within the immune system. The discovery and documentation of epitope data constructs a foundation which new epitope prediction and vaccine development algorithms are built upon.

(2) Gene and genome DNA sequence data: microbial gene DNA sequences have been used for vaccine development through gene cloning and engineering, protein isolation and purification, and vaccine construction. The availability of whole genome sequences for microbial pathogens makes it possible to predict and select protective antigens as vaccine candidates through in silico analysis within the reverse vaccinology strategy.

(3) RNA and protein gene expression data: advanced computational approaches have been developed to analyze high throughput DNA microarray and proteomics data. Detection of RNA and protein gene expression profiles in hosts or pathogens facilitates vaccine design and analysis of hosts' vaccine-induced immune responses. 
(4) Clinical data: statistics methods have routinely been used to analyze data from clinical vaccine trials. Tracking immunization history in computerized registries, modeling the impact of alternative immunization strategies, timely detection of vaccine preventable disease outbreaks, and analyses of vaccine safety concerns are all possible due to analysis of current clinical data. In addition, informatics is also changing postlicensure immunization policy and programs.

(5) Literature data: as the vaccinology field expands and the volume of available data increases, the ability to determine and retrieve useful data is crucial in improving and facilitating further vaccine development and protective immune mechanism research. High throughput literature mining programs are being developed toward these goals.

In addition, mathematical modeling approaches are able to combine both synthetic and real vaccine data to engage specific issues. It is also important to integrate various vaccine-associated data types for more advanced vaccine research and development. To support better vaccine data integration and analysis, manually curated vaccine data have been stored in various vaccine databases (e.g., VIOLIN: http://www.violinet.org/) or represented using a community-based vaccine ontology (VO).

This special issue aspires to become an international forum for researchers to provide testimony regarding new and exciting developments in the field. The first special issue on vaccine informatics includes 20 articles that cover a variety of vaccine research areas. This special issue contains 7 research papers, 5 methodology papers, and 7 review papers. The topics in this issue include vaccine informatics reviews, immunoinformatics analysis of immune epitopes, vaccine design based on immunoinformatics and reverse vaccinology, transcriptomics and proteomics analyses, postlicensure vaccine informatics, vaccine literature mining, and immune database management with potential for vaccine research and development. In addition to the many uses of informatics highlighted by the included articles, we should note that systems biology approaches to vaccines are rapidly emerging. These approaches rely entirely on informatics to analyze large datasets deriving from complex and multidimensional systems.

The field of vaccine informatics has attracted some of the most innovative scientists from the vaccinology, microbiology, immunology, epidemiology, and bioinformatics branches.

\section{Acknowledgments}

As Editors, we are truly privileged to have had many of these individuals contribute to this special issue. It is our hope that the following literature inspires the scientific community to devote their time and efforts into this exciting area. It is through the hard work and dedication of scientists that we are able to develop and utilize cutting edge vaccine informatics methods to advance vaccine $\mathrm{R} \& \mathrm{D}$ and improve immunization programs. We would like to express our sincere gratitude to the contributing authors as well as to the Journal of Biomedicine and Biotechnology.

Yongqun $\mathrm{He}$ Rino Rappuoli

Anne S. De Groot Robert T. Chen 

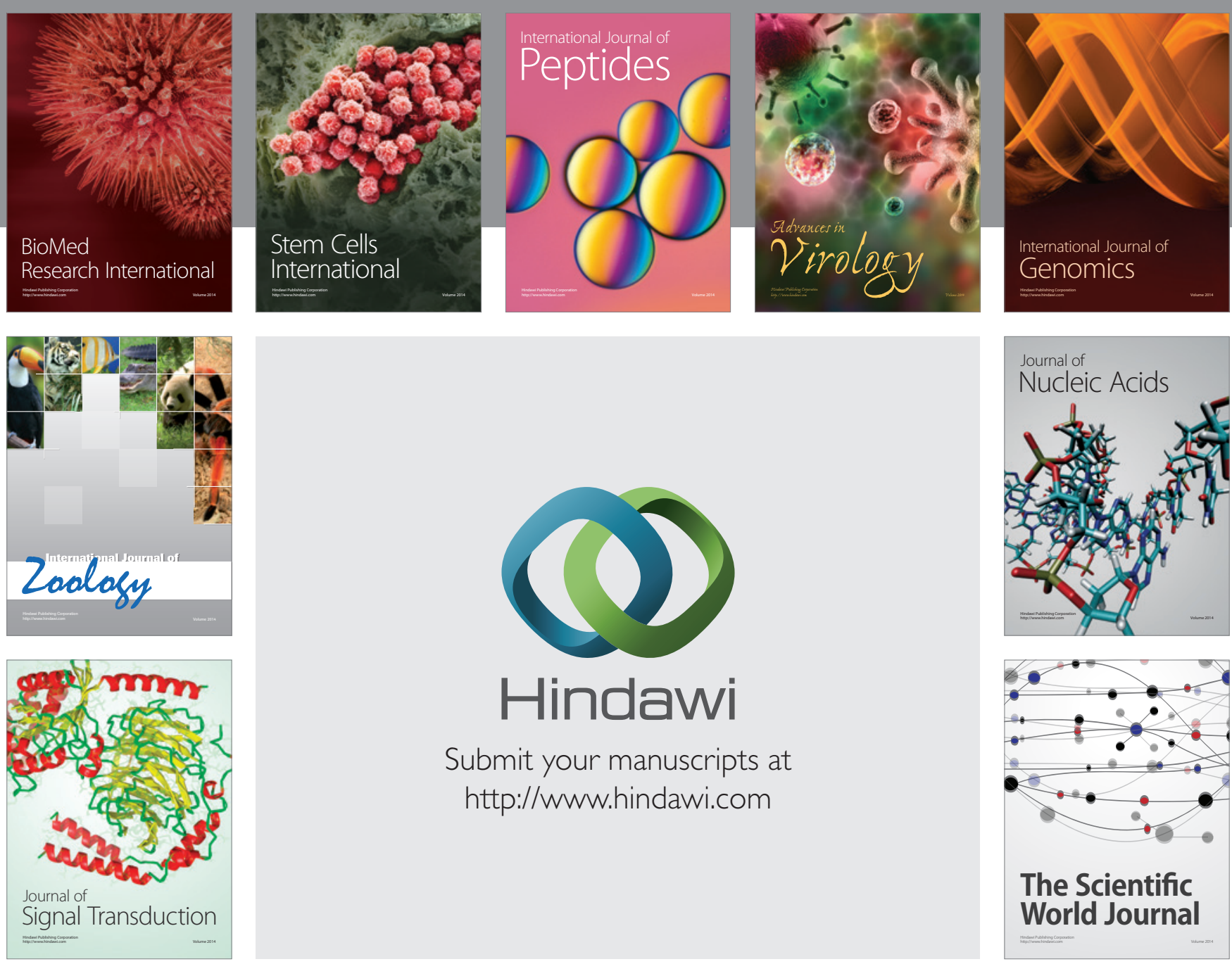

Submit your manuscripts at

http://www.hindawi.com
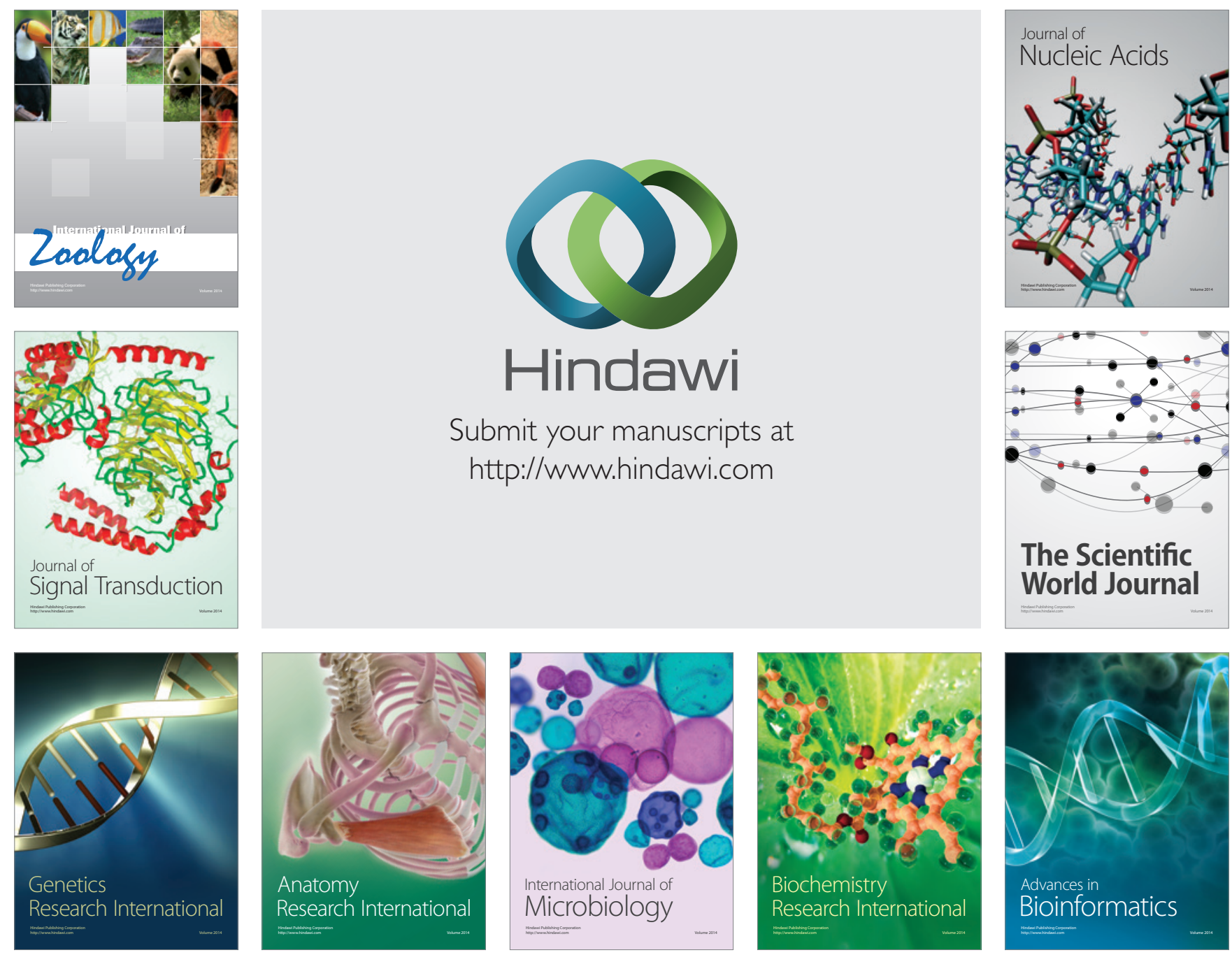

The Scientific World Journal
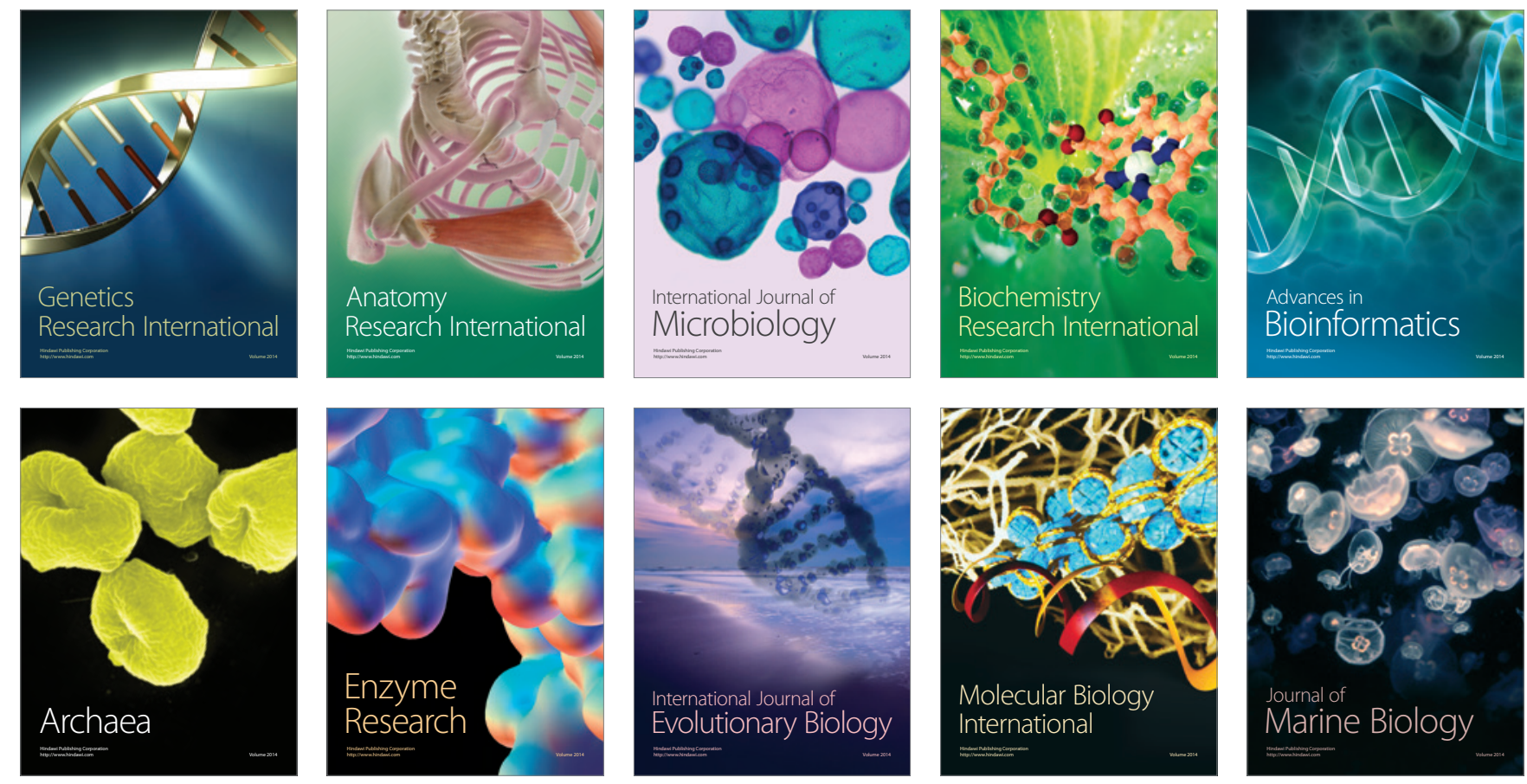